\title{
CONTRIBUIÇÕES DE SIMON STEVIN PARA O DESENVOLVIMENTO DA NOTAÇÃO DECIMAL PARA NÚMEROS NÃO INTEIROS
}

\section{SIMON STEVIN'S CONTRIBUTIONS TO THE DEVELOPMENT OF DECIMAL NOTATION TO NUMBERS NOT INTEGER}

\author{
Thiago Beirigo Lopes ${ }^{1}$; Rosineide de Sousa Jucá ${ }^{2}$, Pedro Franco de Sá ${ }^{3}$
}

\begin{abstract}
RESUMO
Nem sempre os números foram simbolizados como atualmente. Sua representação foi aos poucos sendo alterada e adequada conforme a necessidade e contexto em que o ser humano está imerso. No desenvolvimento de como representar de modo escrito os números decimais, há destaque para o matemático belga Simon Stevin (1548 - 1620). Diante disso, surge a questão norteadora da pesquisa: Qual foi a contribuição de Simon Stevin no processo de desenvolvimento dos números decimais? Em resposta a essa questão, o objetivo da pesquisa apresentada nesse trabalho foi traçar um panorama quanto às contribuições de Simon Stevin por meio de seu livro De Thiende (1585) para o desenvolvimento da escrita de números decimais. Para tanto, foram realizados estudos e traduções do livro original de Stevin (1585) em idioma neerlandês, obtido em formato digital por meio de imagens, e duas traduções desse original. Uma dessas traduções é o livro Disme: the art of tenths or decimall arithmetike realizada por Robert Norton para o idioma inglês com algumas alterações (STEVIN, 1608) e a tradução La Disme realizada por Albert Girard para o idioma francês (STEVIN, 1634), em que este segundo título que foi publicado como um capítulo de seu livro Les Euvres Mathematiques de Simon Stevin de Bruges. Ambas traduções também obtidas em formato digital por meio de imagens da obra original. Nesses livros, são apresentadas as ideias a respeito da notação de números decimais, o desenvolvimento das quatro operações fundamentais, que são adição, subtração, multiplicação e divisão, também as operações de radiciação com base nessa nova notação numérica.
\end{abstract}

Palavras-chave: Números decimais; Operação com números decimais; De Thiende; Simon Stevin; História da Matemática.

1 Doutor em Educação em Ciências e Matemática pela Universidade Federal do Mato Grosso (UFMT/REAMEC). Professor EBTT em Matemática do Instituto Federal de Mato Grosso (IFMT), Confresa, Mato Grosso, Brasil. Endereço para correspondência: Av. Vilmar Fernandes, sn, Bairro Santa Luzia, Confresa, Mato Grosso, Brasil, CEP: 78.652-000. E-mail: thiagobeirigoloes@ yahoo.com.br.

(iD) ORCiD: https://orcid.org/0000-0002-9409-6140.

2 Doutora em Educação em Ciências e Matemática pela Universidade Federal do Mato Grosso (UFMT/REAMEC). Professora da Universidade Estadual do Pará (UEPA), Belém, Pará, Brasil. Endereço para correspondência: Rua do Una, 156, Telégrafo, Belém, Pará, Brasil, CEP: 66050-540. E-mail: rosejuca@mail.com.

(D) ORCiD: https://orcid.org/0000-0002-1386-3388.

${ }^{3}$ Titulação e nome da instituição (SIGLA) em que foi obtida a titulação. Professor Titular da Universidade Estadual do Pará (UEPA), Belém, Pará, Brasil. Endereço para correspondência: Rua do Una, 156, Telégrafo, Belém, Pará, Brasil, CEP: 66050-540. E-mail: pedro.sa@uepa.br.

(iD) ORCiD: https://orcid.org/0000-0002-8986-2787. 
Thiago Beirigo Lopes, Rosineide de Sousa Jucá e Pedro Franco de Sá

Contribuições de Simon Stevin para o desenvolvimento da notação decimal para números não inteiros

\begin{abstract}
The numbers were not always symbolized as they are today. Its representation was gradually changed and adapted according to the need and context in which the human being is immersed. In developing how to represent decimal numbers in writing, the Belgian mathematician Simon Stevin (1548 - 1620) is highlighted. Therefore, the guiding question of the research arises: What was the contribution of Simon Stevin in the process of developing decimal numbers? In response to this question, the aim of the research presented in this work was to outline an overview of Simon Stevin's contributions through his book De Thiende (1585) for the development of writing decimal numbers. To this end, studies and translations of the original book by Stevin (1585) in Dutch language, obtained in digital format through images, and two translations of this original. One of these translations is the book Disme: the art of tenths or decimall arithmetike made by Robert Norton for the English language with some changes (STEVIN, 1608) and the translation La Disme made by Albert Girard for the French language (STEVIN, 1634), in which this second title which was published as a chapter in his book Les Euvres Mathematiques by Simon Stevin de Bruges. Both translations also obtained in digital format through images of the original work. In these books, ideas about the notation of decimal numbers are presented, the development of the four fundamental operations, which are addition, subtraction, multiplication and division, also the radication operations based on this new numerical notation.
\end{abstract}

Keywords: Decimal numbers; Operation with decimal numbers; From Thiende; Simon Stevin; History of Mathematics. 


\section{Introdução}

Desde os primórdios os seres humanos buscam modos de solucionar seus problemas cotidianos e satisfazer suas necessidades. Nesta busca, delineiam suas ações, constroem ferramentas que lhes auxiliam, criam maneiras para se comunicarem desenvolvendo as dimensões da linguagem e, devido à essa necessidade de se aperfeiçoar, elaboram métodos mais eficientes para comunicação dos conhecimentos adquiridos.

Ao imaginar como intervir no ambiente ao seu redor, como registrar suas informações sobre a vida ou suas transações comerciais, os seres humanos criaram meios que, com o decorrer do tempo, permitiram a realização de pesquisas sobre a sua história. No processo de construção do conhecimento matemático, é verossímil que foram necessárias a exploração ou reconstrução de alguns artefatos que permitiram uma nova forma de conhecer e explicar a construção histórica desse conhecimento (OLIVEIRA, 2017).

Nem sempre os números foram simbolizados como nos dias atuais. Sua representação foi aos poucos alterada e adequada conforme necessidade, como a evolução mercantil por exemplo. Para se chegar aos números e à notação numérica atual foi necessário tempo e dedicação de estudiosos de diferentes áreas dentre os quais os matemáticos que, em seu momento da história, contribuíram para seu desenvolvimento.

Diante disso, surge a questão norteadora de pesquisa: "Qual foi a contribuição de Simon Stevin no processo de desenvolvimento dos números decimais? Em resposta à essa questão, o objetivo dessa pesquisa foi o de traçar um panorama quanto às contribuições de Simon Stevin por meio de seu livro De Thiende para o desenvolvimento da escrita de números decimais não inteiros. Para tanto, foram realizados estudos e traduções do livro original de Stevin (1585) em idioma neerlandês, obtido em formato digital por meio de imagens, e as traduções desse original. Uma dessas traduções é Disme: the art of tenths or decimall arithmetike realizada por Robert Norton para o idioma inglês com algumas alterações (STEVIN, 1608) e a tradução La Disme realizada por Albert Girard para o idioma francês (STEVIN, 1634) que foi publicada como um capítulo de seu livro Les Euvres Mathematiques de Simon Stevin de Bruges. Ambas traduções também obtidas em formato digital por meio de imagens.

Para alcançar o objetivo da pesquisa, é apresentado um texto composto por considerações a respeito de De Thiende de Simon Stevin. Em que são apresentadas ideias a respeito da notação de números decimais, o desenvolvimento das quatro operações fundamentais e a radiciação sob essa nova notação numérica. 
Thiago Beirigo Lopes, Rosineide de Sousa Jucá e Pedro Franco de Sá

Contribuições de Simon Stevin para o desenvolvimento da notação decimal para números não inteiros

\section{De Thiende de Simon Stevin}

De acordo com Cajori (1928), antes de 1585 as frações decimais eram utilizadas para os mais diversificados cálculos, principalmente nas práticas sociais. Assim, ainda conforme o autor supracitado, devido aos cálculos longos e trabalhosos das operações com as frações decimais, Stevin sentiu necessidade de criar um modo de fazer essas operações de modo mais ágil e simples. Surgindo assim, o livro De Thiende em 1585. O tratado, segundo Jucá e Sá (2014), teve grande influência na prática comercial, na engenharia e na notação matemática, pois trouxe uma facilidade de cálculos principalmente para os engenheiros da época.

Cajori (1928, p. 314) afirma que o livro “[...] marcou uma compreensão completa da natureza e importância das frações decimais, mas trabalhou sob o peso de uma notação desajeitada". Nela, foram explicadas relações entre as frações decimais, a notação para as representações decimais, regras para as operações aritméticas e suas justificativas. De Thiende é constituído de duas partes: a primeira versa sobre a apresentação da notação sugerida pelo autor e suas quatro definições e a segunda, é composta pelas proposições sobre o procedimento de execução de cada uma das quatro operações fundamentais e, indica que pode ser efetuado qualquer extração de raízes. Ainda é apresentado um apêndice em que são dados exemplos de aplicações dos decimais em vários ramos de atividades como monetário, astronomia, medição de terras, de tapetes e de licor. O próprio livro De Thiende apresenta sua composição, conforme o a

Figura 1. 
Figura 1 - Diagrama de composição do livro De Thiende de Simon Stevin.

\begin{tabular}{|l} 
O Décimo em \\
duas partes.
\end{tabular}$\left\{\begin{array}{l}\text { Definições, como que é }\left\{\begin{array}{l}\text { Décimo, } \\
\text { Parte inteira, } \\
\text { Décimos, centésimos assim por diante. } \\
\text { Números decimais. }\end{array}\right. \\
\text { Operações ou prática de }\left\{\begin{array}{l}\text { Adição, } \\
\text { Subtração, } \\
\text { Multiplicação, } \\
\text { Divisão. }\end{array}\right. \\
\text { Apêndice: } \\
\text { Utilização em alguns ramos de atividade. }\end{array}\right.$

Fonte: Traduzido e adaptado de Stevin (1585).

Na primeira parte anteriormente anunciada, Stevin apresenta quatro definições de sua representação proposta sobre números decimais. A primeira definição busca fazer uma relação proporcional entre valores dos algarismos que compõem um número. $\mathrm{O}$ autor utiliza o exemplo de $2387 \mathrm{em}$ que cada unidade de 8 (que atualmente corresponde à dezena) é o décimo de cada unidade de 7 (que atualmente corresponde à unidade). A segunda definição se refere à parte inteira de sua representação, em que utiliza como exemplo o número 364 que fica 3640. Ou seja, os números com a representação (0) à direita é a parte inteira do número decimal.

A terceira definição dada indica que um décimo de cada parte vinda da unidade da parte inteira, é chamada de décimos, cuja representação é (1), e cada décima parte da unidade dos décimos, é chamada de centésimos, cuja representação é (2), e assim em diante: com cada décima parte da unidade da precedente representação, sempre em ordem, mais uma vez. Originalmente, Simon apresenta os termos eerfte para décimos, tweeden para centésimos, derden para milésimos e vierden para décimos de milésimos. Apresenta o exemplo 3(1)72)5(3)94 em que é composto por 3 décimos, 7 centésimos, 5 milésimos, 9 décimos de milésimos e em fração decimal é $\frac{3}{10}, \frac{7}{100}, \frac{5}{1000}, \frac{9}{10000}$ e que, somados, representam $\frac{3759}{10000}$. A quarta definição indica que os números das segunda e terceira definições são os números decimais. 
A segunda parte de De Thiende inicia com a proposição da adição em que são indicados 3 números decimais: 27(0)8(1)4(3), 37(06(1)7(2)5(3) e 875(0)7(1) 8(3). São descritos os procedimentos de construção e tais números são dispostos conforme a

Figura 2.

Figura 2 - Operação de adição com os números 27(0)8(1)4(2)7(3), 37(0)6(1)7(2)5(3) e 875(0)7(1)8(2)2(3).

\begin{tabular}{|llllll|}
\hline & & 3 & $(1)$ & 2 & $(3)$ \\
& 2 & 7 & 8 & 4 & 7 \\
& 3 & 7 & 6 & 7 & 5 \\
8 & 7 & 5 & 7 & 8 & 2 \\
\hline 9 & 4 & 1 & 3 & 0 & 4 \\
\hline
\end{tabular}

Fonte: Stevin (1585, p. 13).

Para afirmar sua proposição como válida, é realizada uma relação equivalente entre a forma decimal e a forma fracionária em que, ambas as representações, chegam ao mesmo resultado. Assim, os decimais 27(0)8(1)4(2)7(3), 37(0)6(1)7(2)5(3) e 875(0)7(1)8(2)23 são transformados, em notação atual, em $27 \frac{847}{1000}, 37 \frac{675}{1000}$ e $875 \frac{782}{1000}$.

Posteriormente indica que o resultado em forma fracionária é $941 \frac{304}{1000}$, que é representado em forma decimal por 941(0)3(1)0243. Portanto, é concluído ser verdadeira a soma demonstrada. Ainda é indicado uma ressalva de como proceder em caso de faltar alguma casa decimal, como 8(0)5(1)6(2) e 5072) (em que o último necessita do termo para a ordem (1)). É indicado que em seu local deve ser colocado o termo 0(1), para ficar sob a forma 5(0)0(1)72), e pode ser realizada a adição com essa representação. Stevin não faz essa mesma ressalva para as demais proposições, no entanto tal ressalva é imprescindível em todas as demais proposições.

A segunda proposição, da subtração, é apresentada de modo semelhante à proposição da adição. São indicados dois números, 237(0)5(1)7(2)8(3) e 59(0)7(2)93, e é pretendido subtrair o segundo do primeiro. O autor indica que a subtração com esses decimais pode ser feita do mesmo modo que é realizada com os números inteiros. Esse resultado corresponde à 177(0)8(1)2(2)93 quando se volta à notação decimal inicial, conforme 
Thiago Beirigo Lopes, Rosineide de Sousa Jucá e Pedro Franco de Sá

Contribuições de Simon Stevin para o desenvolvimento da notação decimal para números não inteiros

Figura 3. Ainda, demonstra a validade de modo análogo ao demonstrado para a adição com o estabelecimento de relações entre a fração decimal e a notação decimal proposta.

Figura 3 - Operação de subtração com os números 237(0)5(1)7(2)8(3) e 59(0)7(1)4(2)9(3).

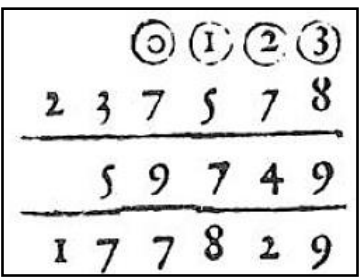

Fonte: Stevin (1585, p. 15).

Na proposição de subtração pode ser percebida uma íntima relação com o modelo usual, em que os números são dispostos de modo que as vírgulas das parcelas da adição necessitam ficar exatamente uma sobre a outra. Em consequência, as casas decimais ficam respectivamente uma sobre a outra e, caso necessário, completa-se as casas faltantes por 0 . Stevin não explicita essa situação na primeira proposição, no entanto esse mesmo método de efetuar como se fossem inteiros e depois voltar para a notação decimal também pode ser estabelecido para a adição.

Para a terceira proposição, da multiplicação, são destacados o número a ser multiplicado 32(0)5(1)72) e o multiplicador 89(0)4(1)62). É indicado que os números dados devem ser multiplicados conforme de multiplicação por números inteiros, desta maneira, resulta o produto 29137122. Agora, para saber quais devem ser as representações dos decimais, as representações à direita de cada número devem ser somadas, que nesse caso são (2) e (2) e somam (4). Desse modo, a primeira representação à direita do resultado deve ser (4) e os demais, da direita para esquerda, são dispostos em ordem contínua. Então o resultado pretendido é 2913(0)7(1)1(2)2(3)2(4) como pode ser verificado na

Figura 4. 
Figura 4 - Operação de multiplicação entre os números 32@5(1)7(2) e 89@4(1)6(2).

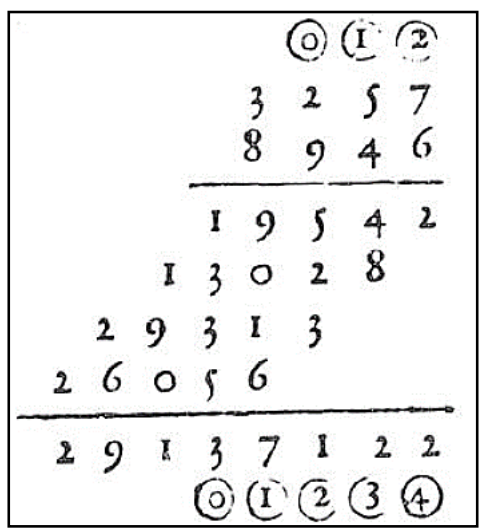

Fonte: Stevin (1585, p. 15).

Como realizado nas duas primeiras proposições, para demonstrar a validade de sua terceira proposição é realizada uma analogia entre as formas de fração decimal e a notação decimal proposta. Em que apresenta 32(0)5(1)7(2) como $32 \frac{57}{100}$ e 89(0)4(1)6(2) como $32 \frac{46}{100}$ e, ao multiplicar as frações decimais, resulta $2913 \frac{7122}{10000}$, que é representado por 2913(0)7(1)12)2(3)2(4) na notação decimal sugerida.

Ainda é explicitado que, para o resultado se deve somar as representações mais à direita de cada fator, assim a multiplicação entre 3(4)7(5)8(6) e 5(1)4(2), em que a soma da última representação à direita de cada número decimal (6) e (2) resulta em (8), serão manipulados conforme descrito na Figura 5.

Figura 5 - Operação de multiplicação entre os números 3(4)7(5)8(6) e 5(1)4(2).

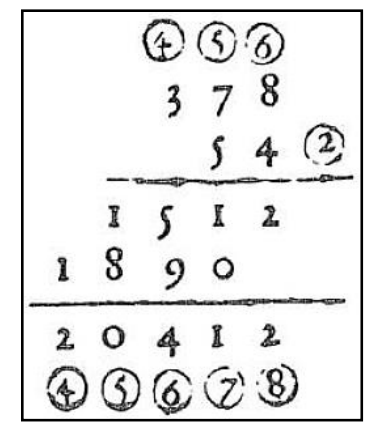

Fonte: Stevin (1585, p. 17).

Em que, em uso atual, é indicado efetuar a multiplicação como se fossem dois números inteiros (sem necessitar de ordem como na adição e subtração), somar as 
quantidades de algarismos à direita da vírgula de cada fator e contar a mesma quantidade no resultado da direita para esquerda e colocar a vírgula.

A quarta e última proposição é referente à divisão em que são dados o dividendo 3(0)4(1)4(2)3(3)5(5) e o divisor 9(1)62). Stevin indica que se deve efetuar a divisão omitindo as representações e procedendo a divisão como se fossem números inteiros, em que resulta o quociente 3587. Então é indicado que a última representação do divisor, que no caso é (2), deve ser subtraída da última representação do dividendo, (5), e isso indica a última representação do último algarismo do quociente, que será (3). As demais representações são dispostas em ordem contínua da direita para a esquerda, portanto o quociente procurado é 3(0)5(1)8(2)7(3).

Como nas proposições anteriores, para demonstrar sua validade foi realizada uma analogia com o mesmo cálculo realizado com notação na forma de fração decimal. Desse modo, o dividendo 3(04(1)4(2)3(3)5(4)2(5) é representado por $3 \frac{44352}{100000}$, o divisor 9(1)62 é representado por $\frac{96}{100}$ e resultam o quociente $3 \frac{587}{1000}$, que é representado na notação proposta como 3(0)5(1)8(2)7(3). Conforme mostrado na Figura 6.

Figura 6 - Operação de divisão em que o dividendo é 3(0)4(1)4(2)3(3)5(4)2(5) e o é divisor 9(1)62).

\begin{tabular}{|c|c|}
\hline$\tilde{y}^{\prime}$ & 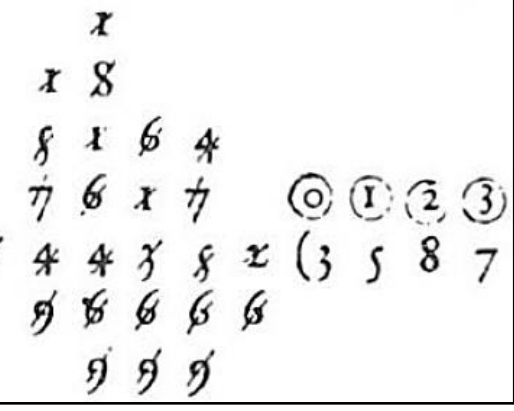 \\
\hline
\end{tabular}

Fonte: Stevin (1585, p. 18).

No entanto, em uma primeira nota, são especificados dois casos particulares que podem ocorrer. O primeiro indica que se a última representação do divisor for maior do que a quantidade de representações do dividendo, é preciso acrescentar quantos algarismos 0 no dividendo forem necessários. É apresentado o exemplo, 72 devem ser divididos por 4(5), em que necessita ser colocada após o 7 a quantidade de três algarismos 0. Portanto fica 7000 e, dividindo-os conforme mencionado resulta o quociente 1750@, conforme é mostrado na Figura 7. 
Thiago Beirigo Lopes, Rosineide de Sousa Jucá e Pedro Franco de Sá

Contribuições de Simon Stevin para o desenvolvimento da notação decimal para números não inteiros

Figura 7 - Operação de divisão em que 7(2) devem ser divididos por 4(5).

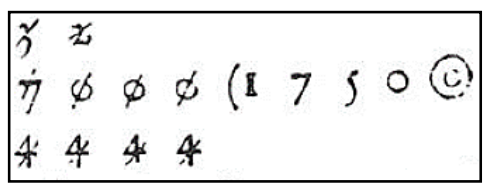

Fonte: Stevin (1585, p. 17).

Ainda com relação à divisão, nesse livro, Stevin (1585) indica que também pode ocorrer do quociente não poder ser expresso por somente números inteiros. Em que cita como exemplo o 4(1) dividido por 32), por meio do qual aparece infinitamente a partir do 3 o resto de $\frac{1}{3}$. Nessa situação é indicado que se pode ir tão longe nas casas decimais quanto for pertinente e são omitidas as demais casas. Então, o quociente buscado é 13(0)3(1) $3 \frac{1}{3}$ (2) (Figura).

Figura 8 - Operação de divisão em que o dividendo é 4(1) e o divisor é 32).

$$
\begin{aligned}
& X X X(1 \quad \text { (j) (1) (2) } \\
& \text { 4. } \varnothing \varnothing \varnothing 000\{1 ; 33
\end{aligned}
$$

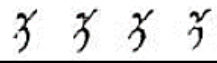

Fonte: Stevin (1585, p. 18).

Diante dessa situação, é demonstrada uma certa frustração ao não ser conseguido um "quociente perfeito", mas o autor destaca que sua intenção no De Thiende é trabalhar todas as operações por meio de números inteiros e para sua utilização em algo posterior. Então destaca que alguns trabalhos em que não são perfeitamente precisos e, nem por isso, tiveram suas contribuições menosprezadas tais como Ptolomeu (90-168) e Johannes Regiomontano (1436-1476) por não descreverem suas Tabelas de Arcos, Cordas ou Senos, com extrema perfeição (como possivelmente poderiam ter feito pela distribuição multinomial), porque essa imperfeição (considerando o alcance e o objetivo dessas Tabelas) é mais conveniente do que a, então, tal perfeição.

Em uma segunda nota, ainda dentro da proposição da divisão, é indicada a possibilidade de extração de todos os tipos de radiciações com essa proposta de números decimais. Como por exemplo, para extrair a raiz quadrada de 5(2)2(3)9(4) efetua-se o cálculo como se houvesse somente números inteiros, ou seja, raiz quadrada de 529 que resulta 23. Então, para o conhecimento da última representação da raiz é a metade da última representação do número dado, que deve ser 2(1)3(2) (Figura ). 
Thiago Beirigo Lopes, Rosineide de Sousa Jucá e Pedro Franco de Sá

Contribuições de Simon Stevin para o desenvolvimento da notação decimal para números não inteiros

Figura 9 - Operação de extração da raiz quadrada de 5(2)2(3)9(4).

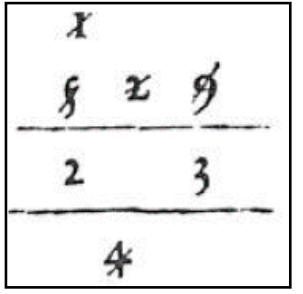

Fonte: Stevin (1585, p. 18).

Ainda destaca que se a última representação dada fosse um número ímpar, a representação do próximo precisa ser adicionado em 1 e então será um número par. Da mesma forma, na extração da raiz cúbica, a terceira parte da última representação dada é a última representação da raiz, e assim para todo tipo de raízes.

A evolução das notações para escrita de números decimais não inteiros, são destacadas por Cajori (1928) e podem ser observadas no Quadro 1.

Quadro 1 - Representação das notações para os decimais em ordem cronológica.

\begin{tabular}{|c|c|c|c|c|}
\hline Autor & Publicação & Lugar & Ano & Notação para 4,683 \\
\hline Simon Stevin & De Thiende & Leyden & 1585 & 4(0)6(1)833 \\
\hline John Napier ${ }^{4}$ & $\begin{array}{l}\text { John Napier's } \\
\text { Rahdologia }\end{array}$ & Edinburgh & 1617 & 4,683 e 4.683 \\
\hline Henry Lyte & $\begin{array}{l}\text { Art of Tens or } \\
\text { Decimall } \\
\text { Arithmetique }\end{array}$ & London & 1619 & $\begin{array}{c}128 \\
4 \mid 683\end{array}$ \\
\hline Joost Bürgi & Progress-Tabulen & Prag & 1620 & $4^{\circ} 683$ e $4_{o} 683$ \\
\hline Henry Briggs & $\begin{array}{l}\text { Arithmetica } \\
\text { logarithmica }\end{array}$ & London & 1624 & $4 \frac{683}{3}$ \\
\hline Adrianus Metius & $\begin{array}{l}\text { Geometriae practicae } \\
\text { pars I et II }\end{array}$ & Lvgdvni & 1625 & $4: 683$ \\
\hline William Oughtred & Clavis mathematicae & London & 1631 & $4 \lcm{683}$ \\
\hline Robert Jager & $\begin{array}{l}\text { Artificial Arithmetick } \\
\text { in Decimals }\end{array}$ & London & 1651 & $\begin{array}{c}* * * \\
4 \mid 683\end{array}$ \\
\hline Francisci à Schooten & $\begin{array}{l}\text { Exercitationvm } \\
\text { mathematicarum } \\
\text { liber primus }\end{array}$ & Leyden & 1657 & 4,6833 \\
\hline John Twysden & $\begin{array}{l}\text { Observationes } \\
\text { eclipsium }\end{array}$ & London & 1659 & $4 . \underline{683}$ e 4.683 \\
\hline
\end{tabular}

\footnotetext{
${ }^{4}$ De acordo com Cajori (1928, p. 195) a notação de Oughtred para decimais deve ter atrasado a adoção geral do ponto decimal ou vírgula proposta por Napier.
} 
Thiago Beirigo Lopes, Rosineide de Sousa Jucá e Pedro Franco de Sá

Contribuições de Simon Stevin para o desenvolvimento da notação decimal para números não inteiros

\begin{tabular}{|l|l|c|c|c|}
\hline Andrea Tacqvet & $\begin{array}{l}\text { Arithmeticae theoria } \\
\text { et praxis }\end{array}$ & Antwerp & 1665 & 4.6 .8 .3 \\
\hline N. Mercator & Logarithmotechnia & $\begin{array}{c}\text { Não } \\
\text { informado }\end{array}$ & 1668 & $4[683$ \\
\hline Joannis Caramvels & $\begin{array}{l}\text { Mathesi8 Biceps. } \\
\text { Vetus, et Nova }\end{array}$ & Companiae & 1670 & $4=683$ \\
\hline Jean Prestet & $\begin{array}{l}\text { Nouveaux elemens } \\
\text { des mathematiques }\end{array}$ & Paris & 1689 & 4683 \\
\hline
\end{tabular}

Fonte: Cajori (1928, p. 211-342).

A rápida difusão das ideias de Stevin (1585) teve a contribuição de traduções de seu livro original em neerlandês para outros idiomas como francês e inglês. A versão inglesa foi realizada por Robert Norton em 1608 (23 anos após a publicação original) sob o título de Disme: the art of tenths or decimall arithmetike (Decimal: a arte dos décimos ou aritmética decimal), no entanto não era uma cópia fiel ao original. O tradutor tomou a liberdade de acrescentar inicialmente os processos de resolução das 4 operações com números inteiros e alterar o exemplo de adição dado originalmente por Stevin, em que troca 37(0)6(1)7(2)5(3) do original por 37(0) 8(1) 5(3) em sua versão inglesa. Desse modo, teve a intenção de facilitar o entendimento para os leigos e facilitar a venda dessa publicação em tua tenda em Londres (STEVIN, 1608). Posteriormente, em 1634 (49 anos após a publicação do original), foi publicado o livro L'Arithmétique (A Aritmética) em que Albert Girard publica uma versão em francês do livro de Stevin sob o título de La Disme (O Decimal). Em que, diferentemente da versão inglesa, faz uma cópia fiel ao original e não altera qualquer componente ou faz acréscimos (STEVIN, 1634).

\section{Considerações Finais}

Com este trabalho foram apresentadas algumas das contribuições de Simon Stevin para a o desenvolvimento da notação dos números decimais, também é destacada a importância do livro De Thiende de Stevin no desenvolvimento dos números decimais. Percebe-se que em um curto espaço de tempo a notação proposta por esse livro foi aprimorado com novas notações que facilitavam a escrita, o cálculo e transpassava algumas limitações. No entanto, é evidente que foi uma proposição que serviu de base alguns estudos. 
Thiago Beirigo Lopes, Rosineide de Sousa Jucá e Pedro Franco de Sá

Contribuições de Simon Stevin para o desenvolvimento da notação decimal para números não inteiros

A importância desse trabalho está no fato desta representação dos números decimais abranger práticas diárias de grande parte da população mundial, tanto na utilização do sistema de medidas padronizadas ou não-padronizadas, transações financeiras e outras situações que dependem da formação sociocultural da população.

Também se compreende que essa a pesquisa não esgotou todas as dimensões abordadas em De Thiende em relação a situações que envolveram a evolução dos números decimais e sua representação (e nem foi esse o objetivo). No entanto, contempla um panorama de sua contribuição na notação e no algoritmo de cálculo.

\section{Referências}

CAJORI, Florian. A history of mathematical notations. Chicago: Open Court Pub, v. $1,1928$.

JUCÁ, Rosineide de Sousa; SÁ, Pedro Franco de. Alguns aspectos históricos dos números decimais. Revista de História da Matemática para Professores, Natal, v. 1, n. 1, p. 29-38, mar. 2014. Disponível em:

http://www.rhmp.com.br/index/index.php/rhmp/article/view/5. Acesso em: 21 set. 2020.

OLIVEIRA, Rosalba Lopes de. Experiências de utilização de artefatos históricos em atividades de ensino. Boletim Cearense de Educação e História da Matemática, Fortaleza, v. 4, n. 11, p. 71-80, 2017. Disponível em:

http://seer.uece.br/?journal=BOCEHM\&page=article\&op=view\&path\%5B \%5D=2627. Acesso em: 20 jan. 2018.

STEVIN, Simon. De Thiende. Leyden: Chriftoffel Plantijn, 1585. Disponível em: http://www.dbnl.org/tekst/stev001thie01_01/stev001thie01_01_scans.pdf. Acesso em: 20 jan. 2018.

STEVIN, Simon. Disme. In: NORTON, Robert (Org.). Disme: the art of tenths or decimall arithmetike. Tradução de Robert Norton. London: Hugh Astley, 1608. Disponível em: https://babel.hathitrust.org/cgi/pt?id=uc1.c042199472; view=1up;seq=1. Acesso em: 20 jan. 2018. (Robert Norton faz uma tradução com acréscimos e algumas alterações do original De Thiende de Simon Stevin).

STEVIN, Simon. La disme. In: GIRARD, Albert (Org.). Les Euvres Mathematiques de Simon Stevin de Bruges. Tradução de Albert Girard. Leinden: [s.n.], 1634. p. 2007213. Disponível em: http://diglib.hab.de/drucke/n-11-2f-helmst/start.htm?image=00220. Acesso em: 28 jan. 2018. (Albert Girard faz uma coletânia dos trabalhos de Simon Stevin em que o capítulo La Disme é uma tradução do original De Thiende).

Recebido em: 09 / 11 / 2020

Aprovado em: 07 / 12 / 2020 